\title{
Technique of Functional and Motility Test: How to Perform Biofeedback for Constipation and Fecal Incontinence
}

\author{
Hyo Jeong Lee, Kee Wook Jung and Seung-Jae Myung* \\ Department of Gastroenterology, Asan Digestive Disease Research Institute, Asan Medical Center, University of Ulsan College of Medicine, \\ Seoul, Korea
}

Biofeedback therapy is an instrument-based learning process centered on operant conditioning. The goal of biofeedback therapy in defecatory disorders is to strengthen the pelvic floor muscles, retrain rectal sensation and coordinate pelvic floor muscles during evacuation. Biofeedback therapy, in a broader sense, includes education, counseling, and diaphragmatic muscle training as well as exercise, sensory, and coordination training. For dyssynergic defecation, biofeedback therapy is a well-known and useful treatment option that had response rates of approximately $70-80 \%$ in randomized controlled trials. Biofeedback therapy for dyssynergic defecation consists of improving the abdominal push effort together with biofeedback technique-guided pelvic floor relaxation followed by simulated defecation and/or sensory training. For fecal incontinence, the results of a randomized controlled trial, which had a response rate of $76 \%$, indicated that biofeedback therapy is useful in selected patients who fail to respond to conservative treatment and that training to enhance rectal discrimination of sensation may be helpful in reducing fecal incontinence. The focus of biofeedback therapy for fecal incontinence is on exercising external sphincter contractions under instant feedback, either alone or synchronously with rectal distension and/or sensory training. Biofeedback therapy is a safe treatment that may produce durable improvement beyond the active treatment period; however, a well-designed study to establish a standard protocol for biofeedback therapy is needed. This review discusses the technique of biofeedback therapy to achieve the goal and clinical outcomes for constipation and fecal incontinence.

(J Neurogastroenterol Motil 2013;19:532-537)

Key Words

Biofeedback; Constipation; Dyssynergic defecation; Fecal incontinence; Treatment outcome

\section{Introduction}

Biofeedback makes physiologic change possible by means of operant conditioning or trial-and-error learning. In this process a response is learned and performed depending on whether that response is followed by reinforcement. ${ }^{1}$ Biofeedback therapy (BFT) is an instrument-based learning process; it uses equipment to record or amplify a patient's bodily activities and then provides feedback to the patient as visual, auditory, or verbal

Received: August 27, 2013 Revised: None Accepted: September 4, 2013

(c) This is an Open Access article distributed under the terms of the Creative Commons Attribution Non-Commercial License (http://creativecommons. org/licenses/by-nc/3.0) which permits unrestricted non-commercial use, distribution, and reproduction in any medium, provided the original work is properly cited.

*Correspondence: Seung-Jae Myung, MD, PhD

Department of Gastroenterology, Asan Medical Center, University of Ulsan College of Medicine, 88, Olympic-ro 43-gil, Songpa-gu, Seoul 138-736, Korea

Tel: +82-2-3010-3917, Fax: +82-2-476-0824, E-mail: sjmyung@amc.seoul.kr

Financial support: This work was supported by the Bio \& Medical Technology Development Program of the National Research Foundation (NRF) fundConflicts of interest: None. ed by the Korean government (MEST) (No. 2011-0019632) and Asan Institute for Life Sciences (No. 2013-261 and 2013-559). 
responses.

The study using biofeedback in the field of gastroenterology was first reported in 1974 and focused on BFT in patients with fecal incontinence $(\mathrm{FI}){ }^{2}$ The first application of BFT for the treatment of chronic constipation due to dyssynergic defecation (DD) was in $1987 .{ }^{3}$ Since then, BFT has been attempted in the treatment of various gastrointestinal disorders, such as functional anorectal pain, irritable bowel syndrome and aerophagia, ${ }^{4}$ but the efficacy of BFT is relatively well-established in the treatment of $\mathrm{DD}$ and FI.

DD is characterized by paradoxical anal contraction, inadequate anal relaxation and/or impaired push effort caused by incoordination of abdominal, rectal and anal muscles. ${ }^{5-7}$ It affects about $40 \%$ of patients with chronic constipation. ${ }^{5}$ FI is the involuntary loss of liquid or solid stool that is a social and hygienic problem. It affects between $1 \%$ and $15 \%$ of adults depending on the definition used. ${ }^{8}$

The goal of BFT in a defecatory disorder is to strengthen the pelvic floor muscles, retrain rectal sensation, and coordinate pelvic floor muscles during evacuation. ${ }^{1,6,9,10}$ This review discusses the technique of performing BFT to achieve the goal and the clinical outcomes of BFT for constipation and FI.

\section{Techniques and Equipment}

BFT equipment consists of a "sensor" to detect the patient's bodily activities (biological signal), an "amplifier" to amplify the detected biological signal by the sensor, and a "conversion device" to transform the biological signal into visual or auditory signals that patients use to gage their response. Visual feedback is provided by observing the changes in pressure or electrical activity on a monitor screen. ${ }^{11}$ Auditory feedback is an audible signal when the biological signal reaches the treatment threshold. Verbal feedback is provided by the therapist by complimenting the patient for performing a correct maneuver or rectifying any errors. ${ }^{11}$

Biofeedback techniques vary considerably among researchers. The most common techniques used to retrain pelvic floor muscles include anorectal manometry to display sphincter pressures or electromyography (EMG) to display electrical muscular activity. ${ }^{6,12}$ In manometry-guided BFT, the manometry probe and a balloon located in the rectum and anal canal provide a visual display of pressure activity throughout the anorectum. ${ }^{6}$ In EMG-guided BFT, surface electrodes are either placed on the external sphincter and buttocks or, more commonly, mounted on an intra-anal sensor. A second EMG channel is used to record electrodes applied to the skin overlying the rectus abdominis muscles. Two-channel EMG provides a visual display of electrical activity of rectus abdominis and external anal sphincter muscles simultaneously. ${ }^{13}$ Home training devices use an EMG home trainer or silicon probe device attached to a hand-held monitor with a liquid crystal display (LCD), which provides visual feedback to the subjects regarding their sphincter response. ${ }^{6,14}$

BFT requires a closed and quiet space and a specialized therapist who offers biofeedback training and education. In addition, a thin catheter with a balloon attached to its tip for balloon expulsion training along with ancillary equipment, i.e., commode, monitor, gloves, lubricating jelly and waterproof paper, are needed.

\section{How to Perform Biofeedback Therapy}

There is no uniform treatment protocol that has been established because a wide variety of biofeedback techniques have been employed with insufficient data to determine the most effective modality. ${ }^{6,9,11,12,15-21}$ BFT is, in a narrow sense, pelvic floor muscle training based on operant conditioning. However, in a broader sense, BFT includes education, counseling, and diaphragmatic muscle training as well as exercise, sensory, and coordination training. We introduce 3 phases of BFT.

\section{Phase 1: Assessment and Education}

Patients are examined by anorectal and colonic function tests before referral for biofeedback. For constipated patients, BFT is applied primarily to patients with DD but it is not helpful in patients with only slow transit constipation. ${ }^{22}$ Therefore, the selection of patients with adequate indications for BFT is important. A therapist checks the gastrointestinal symptom questionnaire, including the stool diary, and reassesses the symptoms of the patient. In the initial phase, the defecation behavior of the patient is evaluated. The patient is asked to sit on a commode with a manometry or EMG probe. Thereafter, the patient is asked to bear down as if to defecate as usual and to contract the external anal sphincter. The EMG pattern during attempted defecation in a patient with dyssynergic defecation is presented in Figure A. The therapist monitors the external appearance of the patient (e.g., breathing pattern, posture and change of abdomen) as well as pressure or electrical activity provided by biofeedback equipment. The therapist discusses the problems and solutions with the patient and prepares treatment plans. 
A
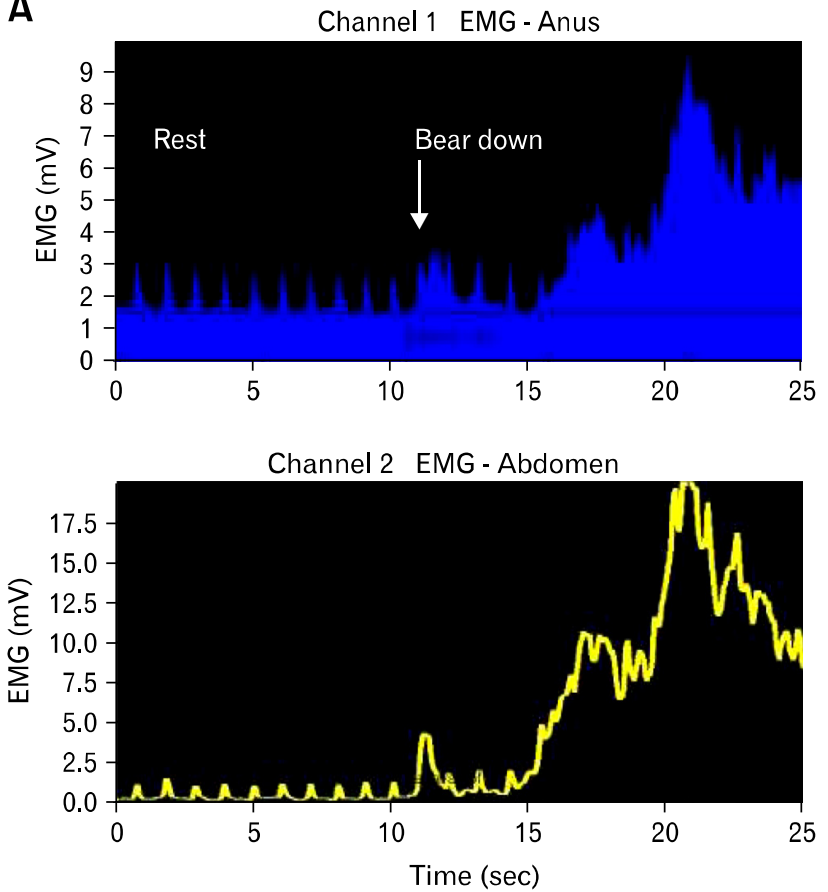

B
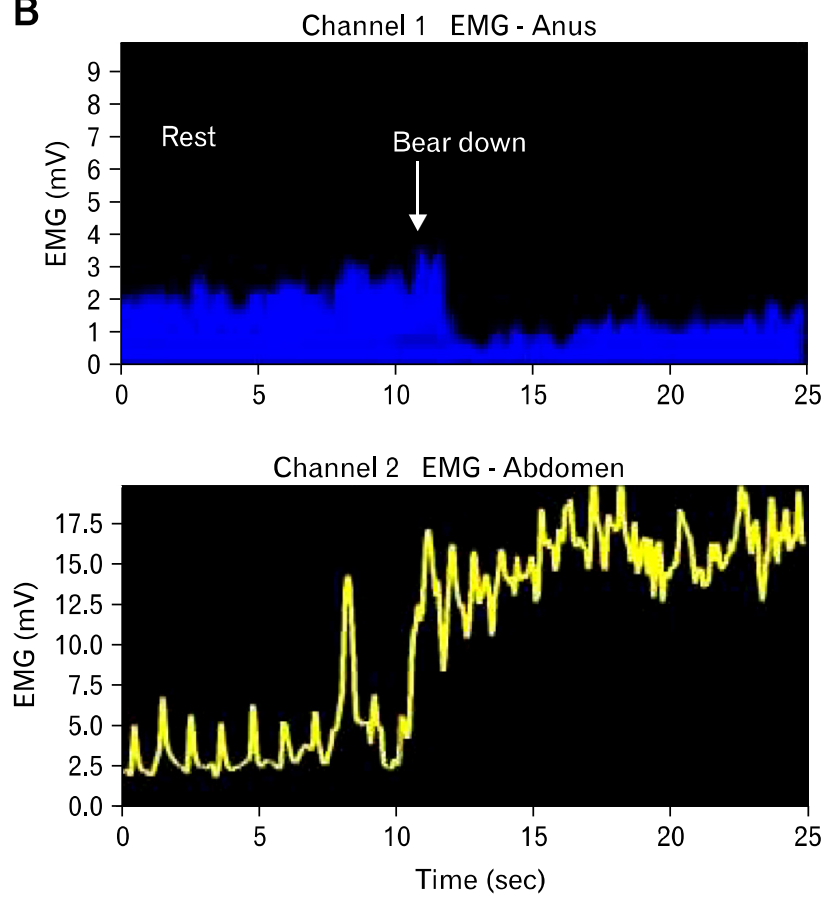

Figure. Electromyography (EMG) pattern changes in a patient with dyssynergic defecation. (A) Before and (B) after biofeedback.

Education and counseling should be performed during the entire BFT period. The objective of BFT and the basic concepts of the anatomy and physiology of the pelvic floor are explained to the patient during the initial session. In addition, the therapist provides life style advice (e.g., dietary habit change and bowel habit information) and basic information about defecatory disorder (e.g., causes, treatment options and use of medication). This can lead to increasing compliance by patients for BFT. Therapist plays an important role; they must communicate and interact well with the patient to establish a supportive relationship. ${ }^{16}$

\section{Phase 2: Active Exercise}

\section{Biofeedback therapy for constipation ${ }^{6,11}$}

BFT for DD consists of improving the abdominal push effort together with biofeedback-guided pelvic floor relaxation followed by simulated defecation training and/or sensory training.

\section{Abdominal push effort (diaphragmatic muscle train-} ing)

First, the patient's posture and breathing technique are corrected. When the patient sits on a commode, the patient should lean forward slightly and keep the legs apart as opposed to keeping them together. The patient is then asked to take a deep diaphragmatic breath to induce proper abdominal pressure for defecation. The patient should repeat exhaling slowly through the mouth and then consciously expand the abdomen while inhaling deeply with the nose.

\section{Rectoanal coordination}

The purpose of this training is to produce a coordinated defecatory movement that consists of an adequate abdominal push effort as reflected by a rise in intrarectal pressure synchronized with anal relaxation. The patient should be seated on a commode with the sensor probe in front of the BFT equipment. The patient is asked to attempt defecation. Instant feedback is provided to the patient regarding the pressure changes in the rectum/anus or abdomen/anus and helps the patient understand and learn quickly. The EMG pattern changes after BFT in a patient with dyssynergic defecation are presented in Figure B. The therapist encourages the patient to pay attention to the pattern of defecation and explains the changes of the pattern to help the patient understand. Approximately 10-15 maneuvers are usually attempted. During the repeated trial, the patient is instructed to change the degree of abdominal push and anal relaxation effort gradually.

\section{Simulated defecation training}

The patient starts to be aware of coordinated defecation through rectoanal coordination training, thereafter, simulated defecation training is performed. The goal of this training is to teach the patient to expel an artificial stool using the correct 
technique. A flexible tube with a $50 \mathrm{~mL}$ water-filled balloon attached to its tip is placed in the rectum in the left lateral position. The patient is asked to sit on a commode and attempt defecation. The patient is taught to relax the pelvic floor muscles and to adopt correct posture and use appropriate breathing techniques. If the patient is unable to expel the balloon, gentle traction is applied to the balloon to supplement the patient's efforts. The maneuver is repeated several times for the patient to learn how to coordinate the defecation maneuver and to expel the balloon.

\section{Rectal sensory training}

Sensory training is applied to constipation patients with rectal hyposensitivity. The goal of this training is to decrease the thresholds of rectal sensory perception by intermittently inflating a balloon in the rectum. A rectal balloon is gradually distended with water, and the patient is asked to report the urge to defecate. Once this threshold volume is determined, the balloon is first inflated to the volume that induces an urge to defecate. Thereafter, for each successive inflation, the balloon volume is decreased in a stepwise manner by about $10 \%$. In manometry-guided BFT, the patient is instructed to observe the monitor and to associate the visual signal provided by the rise in intrarectal pressure with that of any possible rectal sensation during each inflation. Through a process of trial and error, newer thresholds for rectal perception are established.

\section{Biofeedback therapy for fecal incontinence $e^{9,12,18,21}$}

The focus of BFT for FI is on exercising external sphincter contractions under instant feedback, either alone or synchronously with rectal distension and/or sensory training. The patient is first asked to contract the sphincter and is then provided with the information on the degree of external sphincter contraction. To properly contract and relax the external anal sphincter, the patient is instructed to focus on isolation of the muscle.

\section{Strength training}

The goal of this training is to maximize anal sphincter strength and improve speed, duration, and timing of voluntary contractions of the external anal sphincter. The patient should be seated on a commode with the sensor probe and is asked to slowly contract and relax the external anal sphincter. Biofeedback techniques provide information to the patient on anal sphincter pressure or activity regarding the patient's performance. The patient is encouraged to relax abdominal muscles while maximally squeezing the muscles surrounding the anal canal. This maneuver should be performed repeatedly.

\section{Rectal sensory training}

The goal of this training is to achieve a normal level of sen- sory threshold. A flexible tube with a $50 \mathrm{~mL}$ water-filled balloon attached to its tip is placed in the rectum in the left lateral position. Sensory threshold, urge sensation, and maximum tolerated volume are assessed. Patients with rectal hyposensitivity are trained to distinguish and respond to smaller rectal volumes of distension until a normal level of sensory threshold is achieved. Patients with rectal hypersensitivity are trained to tolerate larger volumes by means of progressive distension and urge resistance until a normal level of urge sensation is achieved.

\section{Coordination training}

The goal of this training is to enhance voluntary anal contraction in response to rectal filling, thereby counteracting the rectoanal inhibitory reflex. This is combined with reinforcement of rectal sensitivity and sustained external anal sphincter contractions to improve sphincter strength. The patient is trained to recognize smaller distension volumes and to synchronize and increase external sphincter contractions.

\section{Phase 3: Weaning and Reinforcement}

As the patient starts to confidently control the muscles related to defecation, the frequency of BFT is gradually reduced. There has been considerable variation in BFT protocols, regarding the number, frequency, intensity, and duration of sessions. BFT should be customized for each patient depending on their individual needs. Typically, 1hour per session, bi-weekly training, and a total of four to six training sessions are performed in several randomized controlled trials. ${ }^{6,23,24}$ After completion of intensive training, periodic reinforcements at 6 weeks, 3 months, 6 months and 12 months may provide additional benefit and also improve the long-term outcome in patients with DD. ${ }^{6,24}$

\section{Utility of Biofeedback Therapy}

\section{Efficacy of Biofeedback Therapy for Constipation}

BFT is the most effective treatment for DD. ${ }^{7,14}$ The symptomatic improvement rate has varied between $44 \%$ and $100 \%$ in randomized controlled trials of adults with DD. ${ }^{6}$ All of these studies have concluded that BFT is superior to controlled treatment approaches, such as diet, exercise, laxatives, sham feedback, ${ }^{23}$ use of polyethylene glycol, ${ }^{22}$ and placebo or diazepam ${ }^{13}$ in the management of patients with DD. A meta-analysis revealed that BFT is more than three times as effective as non-BFT, but equal efficacy of EMG-guided BFT to other applications was al- 
so shown. ${ }^{25}$ BFT for DD also improves global symptoms and normalizes colon transit time as well as the dyssynergic pattern of defecation and constipation symptoms. ${ }^{13,22-24,26}$ However, the impact of BFT on quality of life or psychological state had not been clearly assessed. ${ }^{16}$ BFT has been shown to provide sustained improvement of bowel symptoms and anorectal function for up to 2 years. $^{24,26,27}$ The American Gastroenterological Association (AGA) and the Asian Neurogastroenterology and Motility Association guidelines strongly recommend BFT for the management of DD. ${ }^{7,16}$

\section{Efficacy of Biofeedback Therapy for Fecal Incontinence}

BFT for FI was an established method for treating FI patients in the era prior to randomized controlled trials. ${ }^{28,29}$ However, a randomized controlled trial of BFT for FI in 2003 suggested that there was no difference between different conservative interventions and that adding exercises or biofeedback or both did not enhance the outcomes of conservative management. ${ }^{30}$ Moreover, a meta-analysis indicated that BFT in FI was equally as effective as non-BFT. ${ }^{25}$ The role and proper indication of BFT in the management of FI have not been established. However, a recent randomized control study that enrolled only those who failed to respond to conservative treatment showed that BFT had a $76 \%$ response rate and was more effective than exercise alone. ${ }^{21}$ In addition, rectal balloon training to enhance rectal discrimination of sensation may be helpful in reducing FI. ${ }^{18}$ The benefits of BFT last at least 12 months. ${ }^{4,21}$ Guidelines issued by AGA recommend BFT for patients with weak sphincters and/or impaired rectal sensation. ${ }^{31}$ One small study reported that BFT was ineffective in neurogenic FI. ${ }^{32}$ Several recent studies demonstrated that the combination of electrical stimulation with BFT is superior to BFT alone. ${ }^{33,34}$

In summary, although the evidence for BFT in the management of FI is still lacking, BFT for FI may be useful in selected patients who fail to respond to conservative treatment. Additionally, training to enhance rectal discrimination of sensation may be helpful in reducing FI. Further well-designed, randomized, controlled trials are required to validate outcome measures and long-term follow-up.

\section{Limitations of Biofeedback Therapy}

BFT requires proper equipment, a private and quiet space, and a well-trained therapist. In clinical practice, it may be difficult for a patient to obtain BFT because of logistical (no facility) or financial reasons. A recent study indicated that only $44-48 \%$ of patients who were recommended BFT actually underwent $\mathrm{BFT}^{10}$ because of the lack of insurance coverage, distance to local treatment facilities, and acute medical issues taking precedence. Moreover, BFT is time-consuming, and repeated training is required; hence, a willingness to participate is important for successful BFT. ${ }^{35}$ Physicians and therapists need to continuously motivate patients through education and counseling.

Another limitation is that there is no established standard protocol for BFT. Further studies should assess the most effective training program to be applied to each component of BFT, e.g., education, life style advice, diaphragmatic muscle training, exercise, sensory and coordination training. An assessment of the impact of BFT on quality of life, psychological state, or economic analyses is also needed.

\section{Conclusions}

BFT is an established treatment for DD and is useful in FI patients who fail to respond to conservative treatment. Moreover, BFT is a safe treatment that may produce durable improvement beyond the active treatment period. There is a need for a well-designed study to establish a standard protocol for BFT.

\section{References}

1. Jung KW, Yang DH, Myung SJ. Biofeedback therapy. In: Ramachandran VS, ed. The encyclopedia of human behavior. Volume 1. 2nd ed. San Diego: Academic Press 2012:344-347.

2. Engel BT, Nikoomanesh P, Schuster MM. Operant conditioning of rectosphincteric responses in the treatment of fecal incontinence. $\mathrm{N}$ Engl J Med 1974;290:646-649.

3. Bleijenberg G, Kuijpers HC. Treatment of the spastic pelvic floor syndrome with biofeedback. Dis Colon Rectum 1987;30:108-111.

4. Chiarioni G, Whitehead WE. The role of biofeedback in the treatment of gastrointestinal disorders. Nat Clin Pract Gastroenterol Hepatol 2008;5:371-382.

5. Rao SS. Dyssynergic defecation and biofeedback therapy. Gastroenterol Clin North Am 2008;37:569-586, viii.

6. Rao SS. Biofeedback therapy for constipation in adults. Best Pract Res Clin Gastroenterol 2011;25:159-166.

7. Bharucha AE, Pemberton JH, Locke GR 3rd. American Gastroenterological Association technical review on constipation. Gastroenterology 2013;144:218-238.

8. Norton C. Fecal incontinence and biofeedback therapy. Gastroenterol Clin North Am 2008;37:587-604, viii.

9. Norton C, Cody JD. Biofeedback and/or sphincter exercises for the treatment of faecal incontinence in adults. Cochrane Database Syst Rev 2012;7:CD002111. 
10. Jodorkovsky D, Dunbar KB, Gearhart SL, Stein EM, Clarke JO. Biofeedback therapy for defecatory dysfunction: "real life" experience. J Clin Gastroenterol 2013;47:252-255.

11. Rao SS, Welcher KD, Pelsang RE. Effects of biofeedback therapy on anorectal function in obstructive defecation. Dig Dis Sci 1997;42: 2197-2205.

12. Jorge JM, Habr-Gama A, Wexner SD. Biofeedback therapy in the colon and rectal practice. Appl Psychophysiol Biofeedback 2003; 28:47-61.

13. Heymen S, Scarlett Y, Jones K, Ringel Y, Drossman D, Whitehead WE. Randomized, controlled trial shows biofeedback to be superior to alternative treatments for patients with pelvic floor dyssynergia-type constipation. Dis Colon Rectum 2007;50:428-441.

14. Schey R, Cromwell J, Rao SS. Medical and surgical management of pelvic floor disorders affecting defecation. Am J Gastroenterol 2012; 107:1624-1633.

15. Park SK, Myung SJ, Jung KW, et al. Biofeedback therapy in constipated, female patients and caused by radical hysterectomy or vaginal delivery. J Gastroenterol Hepatol 2013;28:1133-1140.

16. Gwee KA, Ghoshal UC, Gonlachanvit S, et al. Primary care management of chronic constipation in Asia: the ANMA chronic constipation tool. J Neurogastroenterol Motil 2013;19:149-160.

17. Ahn JY, Myung SJ, Jung KW, et al. Effect of biofeedback therapy in constipation according to rectal sensation. Gut Liver 2013;7:157162.

18. Bols E, Berghmans B, de Bie R, et al. Rectal balloon training as add-on therapy to pelvic floor muscle training in adults with fecal incontinence: a randomized controlled trial. Neurourol Urodyn 2012; 31:132-138.

19. Yang DH, Myung SJ, Jung KW, et al. Anorectal function and the effect of biofeedback therapy in ambulatory spinal cord disease patients having constipation. Scand J Gastroenterol 2010;45:12811288.

20. Shin JK, Cheon JH, Kim ES, et al. Predictive capability of anorectal physiologic tests for unfavorable outcomes following biofeedback therapy in dyssynergic defecation. J Korean Med Sci 2010;25:10601065 .

21. Heymen S, Scarlett Y, Jones K, Ringel Y, Drossman D, Whitehead WE. Randomized controlled trial shows biofeedback to be superior to pelvic floor exercises for fecal incontinence. Dis Colon Rectum 2009;52:1730-1737.

22. Chiarioni G, Whitehead WE, Pezza V, Morelli A, Bassotti G. Biofeedback is superior to laxatives for normal transit constipation due to pelvic floor dyssynergia. Gastroenterology 2006;130:657-664.

23. Rao SS, Seaton K, Miller M, et al. Randomized controlled trial of biofeedback, sham feedback, and standard therapy for dyssynergic defecation. Clin Gastroenterol Hepatol 2007;5:331-338.

24. Rao SS, Valestin J, Brown CK, Zimmerman B, Schulze K. Longterm efficacy of biofeedback therapy for dyssynergic defecation: randomized controlled trial. Am J Gastroenterol 2010;105:890-896.

25. Enck P, Van der Voort IR, Klosterhalfen S. Biofeedback therapy in fecal incontinence and constipation. Neurogastroenterol Motil 2009; 21:1133-1141.

26. Chiarioni G, Salandini L, Whitehead WE. Biofeedback benefits only patients with outlet dysfunction, not patients with isolated slow transit constipation. Gastroenterology 2005;129:86-97.

27. Lee BH, Kim N, Kang SB, et al. The Long-term Clinical Efficacy of Biofeedback Therapy for Patients With Constipation or Fecal Incontinence. J Neurogastroenterol Motil 2010;16:177-185.

28. Heymen S, Jones KR, Ringel Y, Scarlett Y, Whitehead WE. Biofeedback treatment of fecal incontinence: a critical review. Dis Colon Rectum 2001;44:728-736.

29. Whitehead WE, Wald A, Norton NJ. Treatment options for fecal incontinence. Dis Colon Rectum 2001;44:131-142; discussion 142144.

30. Norton C, Chelvanayagam S, Wilson-Barnett J, Redfern S, Kamm MA. Randomized controlled trial of biofeedback for fecal incontinence. Gastroenterology 2003;125:1320-1329.

31. Rao SS; American College of Gastroenterology Practice Parameters Committee. Diagnosis and management of fecal incontinence. American College of Gastroenterology Practice Parameters Committee. Am J Gastroenterol 2004;99:1585-1604.

32. van Tets WF, Kuijpers JH, Bleijenberg G. Biofeedback treatment is ineffective in neurogenic fecal incontinence. Dis Colon Rectoum 1996;39:992-994.

33. Schwandner T, Hemmelmann C, Heimerl T, et al. Triple-target treatment versus low-frequency electrostimulation for anal incontinence: a randomized, controlled trial. Dtsch Arztebl Int 2011; 108:653-660.

34. Vonthein R, Heimerl T, Schwandner T, Ziegler A. Electrical stimulation and biofeedback for the treatment of fecal incontinence: a systematic review. Int J Colorectal Dis Online First: 31 Jul 2013. doi: 10.1007/s00384-013-1739-0

35. Shim LS, Jones M, Prott GM, Morris LI, Kellow JE, Malcolm A. Predictors of outcome of anorectal biofeedback therapy in patients with constipation. Aliment Pharmacol Ther 2011;33:1245-1251. 